\title{
Author Correction: Surface passivation of perovskite film for efficient solar cells
}

Qi Jiang, Yang Zhao, Xingwang Zhang $\mathbb{D}^{\mathbb{D}}$, Xiaolei Yang, Yong Chen, Zema Chu, Qiufeng Ye, Xingxing Li, Zhigang Yin and Jingbi You iD

Correction to: Nature Photonics https://doi.org/10.1038/s41566-019-0398-2, published online 1 April 2019.

In the version of this Article originally published online, in Fig. 1b, the chemical structures of phenylethylamine and formamidine had incorrect numbers of hydrogen atoms. In Fig. $4 \mathrm{a}$, in the legend, the black and red lines were reversed; the black line should have been labelled 'Without PEAI fitting' and the red line 'With PEAI fitting. These errors have now been corrected in all versions.

Original

b

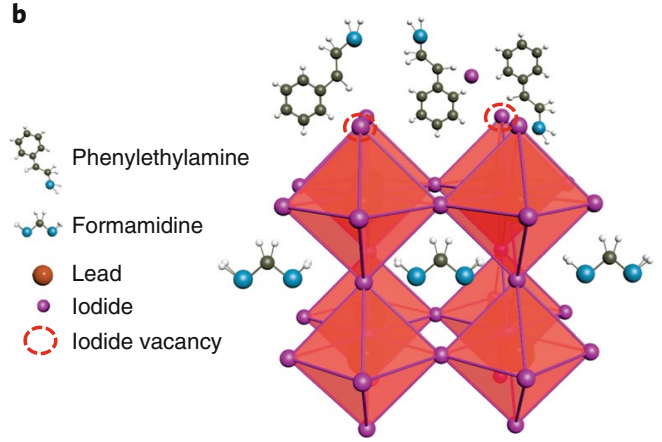

Corrected

b

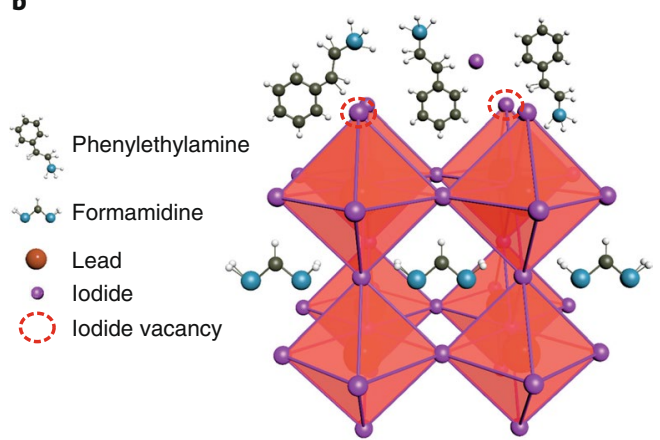

Fig. 1b | Original and corrected.

Published online: 8 May 2019

https://doi.org/10.1038/s41566-019-0462-y 